\title{
Cashing in your chips: speculation on the future of diagnostic laboratories in the era of DNA chips
}

The technology, workflow, turnover time-to-report and organisation of diagnostic bacteriology laboratories have changed little since the end of the 19th century. Computers, anaerobe and safety cabinets, and mediamaking and blood-culture machines have arrived over the past 30 years. However, these are adjuncts, improving quality and safety, and allowing higher throughput with fewer staff, but making little difference to the laboratory organisation, and having little impact on the turnover time from specimen arrival to final report. While there are clear difficulties in automating and speeding up diagnostic work, it seems unlikely that advanced technology would not have more fundamental changes to offer. We have been exploring the possible consequences of the marriage of two new technologies - molecular methods and electronic chips - on the future of medical microbiology and laboratory organisation. This has led us to believe that a hand-held instrument capable of bedside diagnosis of most infectious diseases - viral, bacterial, fungal or protozoal - may become available within the next decade. Here, we outline the current techniques that might contribute to such instruments, and speculate on their future development and effects upon diagnostic microbiology services.

One component of the new technology is the electronic chip. The demands for micro-miniaturised components from the computer industry have driven rapid development of micro-fabrication processes. Enormously complex three-dimensional designs can be fabricated with individual components $<1 \mu \mathrm{m}$ in size. With the current wide range of precision masking, etching and deposition methods, large, multi-layered chips can be produced with exquisitely fine microstructure. The main costs involved are in the chip design and the fabrication equipment; materials costs are small and high-output mass production can be readily achieved. Although the main thrust has been to produce microminiaturised electronic components, micro-mechanical and micro-analytical devices have been manufactured. These include micro-pumps, motion sensors and gasliquid chromatographs, and progress is being made towards the design of mass spectrometers and other complex analytical devices on single chips. Chip-based miniaturisation is an essential prerequisite for a portable hand-held device to diagnose infectious disease.

The new technology of nucleic acid amplification and detection is another essential component. Molecular methods for detection of infectious agents follow a common approach, despite wide differences in detailed methodology (Fig. 1). In the initial 'clean-up' step, the nucleic acids are extracted from the specimen, and nucleases and other factors that might interfere with amplification or detection are neutralised or removed. The next stage is to amplify a specific portion of the nucleic acid many-fold by isothermal methods (e.g., nucleic acid sequence-based amplification; NASBA) or thermocycling reactions (e.g., PCR). This substitutes for the 'amplification' of viral or bacterial populations that occurs during conventional in-vitro propagation, but brings concentrations of amplified nucleic acids to detectable levels in $<2 \mathrm{~h}$. Finally, the product is detected and identified, often by hybridisation with a specific probe.

Amplification/probe-based diagnostic techniques for pathogens are well developed, but are highly specific for a single target. Development has centred on amplification, detection and identification of sequences specific to individual pathogens, e.g., Mycobacterium tuberculosis, human immunodeficiency virus and hepatitis $\mathrm{C}$ virus. These molecular methods can each detect their individual target pathogen rapidly, efficiently and with high sensitivity, but there is no single method that is the molecular equivalent of conventional culture on rich medium to detect a wide range of bacterial pathogens. The approach has not yet been generalised to detect the presence of, and identify, any infectious agent - viral, bacterial, fungal or protozoal - with a single assay. The hypothesis presented here is that the combination of chip and molecular technologies may well offer a rapid, generalised approach to diagnosis of infectious diseases.

Chip and molecular technologies are now coming together. Perhaps the most publicised recent advance has been the development of micro-arrays. These represent the next step in a miniaturisation process that has moved many microbiological tests from test tubes to ever-smaller multi-well plates. Tiny spots of nucleic acids, proteins or other biological molecules are deposited or synthesised on a surface, usually on glass. For example, arrays of up to 500000 distinct nucleic acid spots can be synthesised in situ or applied on a space smaller than a microscope slide.

These arrays currently find their main use in investiga- 


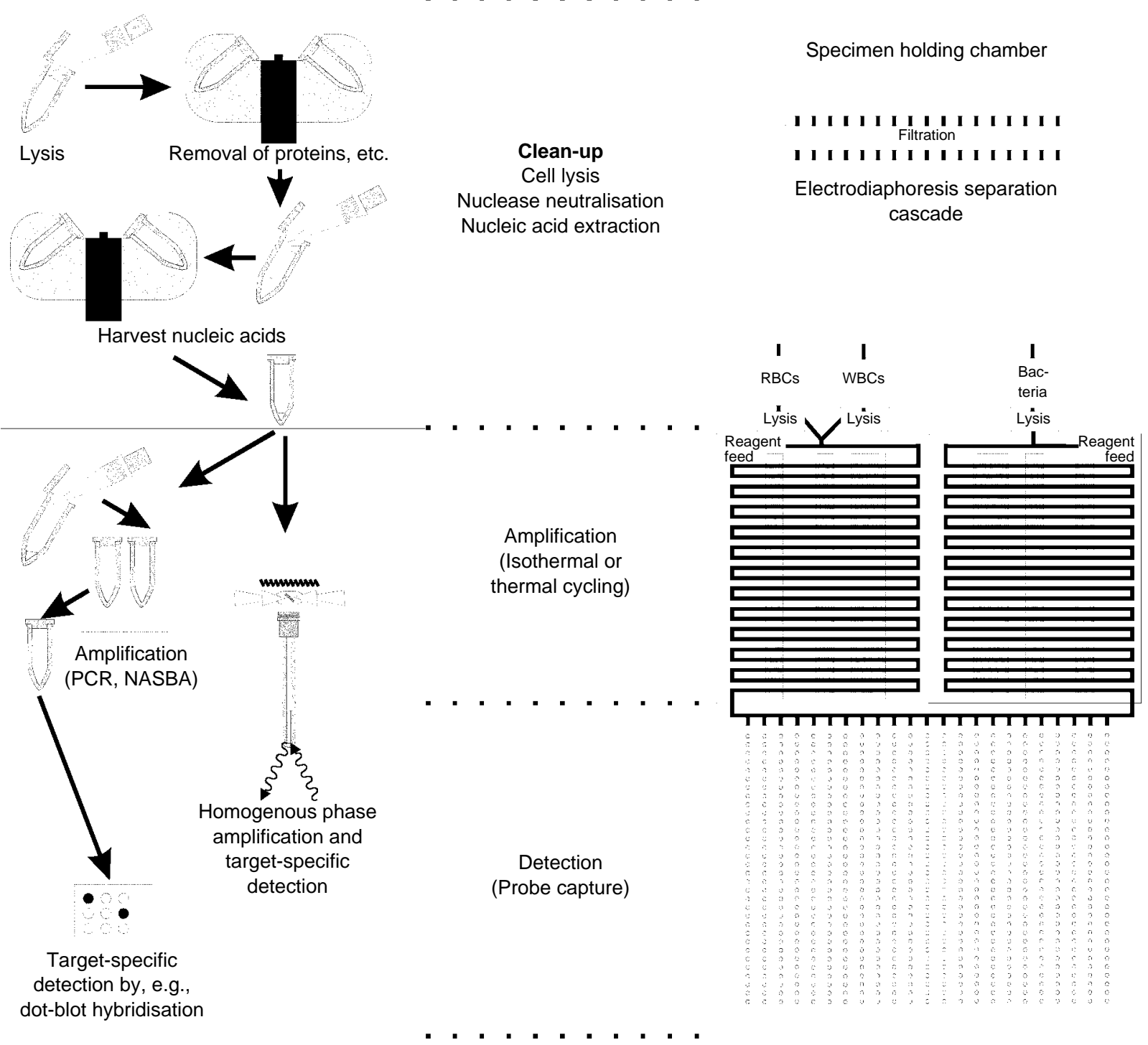

Fig. 1. Molecular methods as applied currently (left), and as they might be applied exploiting chip technology (right). The hypothetical chip utilises electrodiaphoresis to separate intact host cells (WBCs, white blood cells; RBCs, red blood cells) and bacteria before lysis, PCR thermocycling for amplification and a micro-array for detection.

tions of gene expression, where mRNA is extracted and amplified with marker bases that label products from the control and test preparations with different fluorescent dyes. The products are then hybridised on to an array of bound nucleic acid spots, each representing a unique sequence from a distinct gene of interest. After washing, the spots are viewed under UV light by computerised confocal microscopy. The relative proportions of colour from the two dyes indicate whether mRNA synthesis from the specific gene that corresponds to the spot has been upregulated, down-regulated or remained the same as in the control. The fluorescence detection system requires bulky array reading instruments, but there is now considerable interest in systems that respond electronically to hybridisation of test material to an array spot, sending a signal through electronics fabricated in the substrate underlying the spots [1]. Other applications of micro-arrays include gene sequencing, detection of single nucleotide polymorphisms and assessment of drug susceptibility and mode of action [2-8]. Clearly, there are many further potential applications for a device that offers specific detection for such large numbers of amplified products, including some of particular interest in medical microbiology.

However, the DNA chip is not the only micro-device that may be pertinent to micro-miniaturised molecular methods. The first step in amplification/probe detection methods (Fig. 1) is specimen clean-up. This is probably the least well-developed portion of the technology required for a diagnostic device. One approach might be to simply lyse all cells present, and separate the extracted nucleic acids with a centrifugation step, external or on-chip filtration, on-chip microcapilliary electrophoresis [9], or electrophoretic trapping [10]. There may be problems with excess viscosity, or with generalised DNA amplification (see below) in speci- 
mens rich in host-derived DNA, e.g., pus. These could be avoided by DNAase digestion, followed by amplification of RNA rather than DNA, or in some way biasing the generalised amplification to minimise amplification of host nucleic acids.

A more selective approach would be to sort and separate the incoming particles from the specimen, selecting and concentrating intact bacterial and eukaryotic cells (possibly containing viral and other intracellular pathogens) for lysis. One study made use of micro-fabricated silicon microfilter chambers for cell isolation and preparation of DNA [11]. A similar approach could perhaps be used as the first stage in analysis for cell-associated pathogens. One of the most promising lines of development exploits an effect called electrodiaphoresis. In this, particles in a suspension are manipulated in an electrical field and move according to the relative charge properties of the particle and the suspending fluid. Sophisticated separations can be achieved with microscopic travellingcharge electrodiaphoresis guides. Effectively, the particles are directed through channels analogous to conveyor belts, roundabouts and railway points, and highly specific separation can be achieved. Escherichia coli has been separated from a mixture with red blood cells on to charged electrodes and lysed with a high voltage pulse on a chip device [12]. The extracted DNA was then transferred to an array, and the bacterial component was detected by binding with speciesspecific oligonucleotide spots. This simple approach was successful, proving the potential for clean-up and lysis, but more sophisticated processing is likely to be required for the general run of diagnostic specimens. With suitable suspending fluids and micro-sensors to detect and respond to particle size, shape and movement, electrodiaphoresis devices could be developed to shunt red blood cells, leucocytes and bacteria from a specimen into separate compartments for voltage pulse lysis and release of nucleic acids. It may also be possible to manipulate intact viruses in clinical samples by electrodiaphoresis [13].

The amplification step has received far more attention. Almost all the approaches to nucleic acid amplification have been investigated in chip format and in some cases have been combined with cell isolation and nucleic acid preparation [11]. At least theoretically, isothermal amplification techniques such as NASBA would fit readily into the chip-based array technology and are particularly useful for analysis of RNA molecules (e.g., genomic viral RNA, rRNA and mRNA), as reviewed recently [14]. Many diagnostic laboratories now employ tube-based $\mathrm{PCR} /$ probe-based assays for specific pathogens. A continuous flow PCR chip [15] illustrates the level of microstructure that can be obtained in these micro-devices. The channel where amplification occurs is $90 \mu \mathrm{m}$ wide, $40 \mu \mathrm{m}$ deep and $c$. $2 \mathrm{~m}$ long. This winds back and forth across three temperature-controlled areas held at appropriate melt- ing, hybridisation and extension temperatures, to produce the thermal cycling required. This chip will amplify target DNA sequences to detectable levels in 2 min. With suitable channel surfaces and continuous elution, residual reaction products can be washed out rapidly, and the device re-used for another amplification reaction within a short period.

Chip-based nucleic acid amplification technology is well advanced, but the vision of a chip that can perform as the molecular analogue of the bacteriologist's blood agar plate requires multiplex amplification with a range of primers, or general amplification of nucleic acids from all pathogens present in the specimen. This is much more difficult, but procedures for multiplex or more generalised amplification of all DNA or RNA in a specimen are becoming available. Amplification with degenerate primers could be undertaken as described for random PCR of the human genome [16], where generalised amplification was followed by a second, multiplex PCR of the amplified material to identify relevant deletions. An amplification procedure with an inherent bias against amplification of mammalian nucleic acids would avoid specificity and sensitivity problems at the detection stage. There is no reason to believe that 'general' amplification procedures will not develop further, nor that these techniques, whether based on PCR, NASBA or other methods, will present any more difficulty in micro-miniaturisation than the single-product amplification procedures discussed above.

The final step is detection and identification, where array chip technology will clearly have a role. There are roughly 1000 bacterial species, 100 fungal and protozoal parasites, and perhaps 1000 viruses that are known pathogens of man and farm animals. It appears that many of these have unique highly conserved sequences that could be amplified with a comparatively small range of primers. The conserved sequences of $16 \mathrm{~S}$ and 18S rRNA appear ideal candidates for detection and identification at species level in bacterial and eukaryotic pathogens, and two pairs of universal primers might be sufficient to amplify these. The viruses present greater problems, but where groupspecific genes have been identified these could be exploited to limit the range of primers required. In any case, selection of specific (possibly multiplexed) or 'universal' primers could be aimed at the most likely targets in a particular biological sample. Specific detection of a mere 2100 distinct and unique sequences is well within the capabilities of current chip technology, leaving a large spare capacity that might be used for other purposes.

Detection of resistance to antimicrobial agents would be a clear candidate for use of the spare capacity of a micro-array. The gains from rapid bedside detection of infectious agents would soon be viewed as minor if each specimen also had to be cultured to obtain isolates 
for susceptibility testing. This, and the inevitable opportunities for mismatching of molecular and culture results would provide clear encouragement to develop a concurrent capability for assessment of antimicrobial susceptibilities in the chip-based system. At this stage the number of primers required for amplification of sequences associated with resistance may present difficulties, but, again, the number of unique specific sequences characteristic of susceptibility or resistance to antimicrobial agents may be sufficiently small to allow advances in molecular technology to cope. There would be little problem in terms of specific detection capacity with current technology; the species identification sequences would occupy $<1 \%$ of the available capacity. 'Negative' characters, such as point mutations in the rpoB gene of $M$. tuberculosis conferring rifampicin resistance, can already be detected with line assays, direct sequencing or hybridisation on a moderate density array $[17,18]$. 'Positive' characters, such as production of TEM $\beta$-lactamases, could be detected by amplification and hybridisation of unique sequences. Mutational scanning of PCR products with subtractive oligonucleotide hybridisation analysis has been reported for detection of mutations in the human p53 tumour-suppressor gene [8]. The approach makes use of sensor-chip immobilised counterparts to the probes used in the hybridisation reaction, an approach that could readily be applied to detection of antimicrobial resistance mutations. The problem of differential analysis of expressed versus unexpressed resistance genes might be solved by amplifying RNA rather than DNA, which would fit well with detection of RNA genome viruses, mRNA- and rRNA-specific sequences.

Finally, further reserve detection capacity might be used in epidemiological typing, an important and expanding area which seems ideally suited to chipbased technologies [19]. The molecular methods for detection of antimicrobial resistance could provide useful data on the epidemiology of resistance genes and limited data for strain discrimination. However, this alone may have insufficient discriminatory power. One alternative approach might be to search for a sequence universally present in, for example, bacteria, with highly conserved terminal regions for priming, but showing high inter-strain variation in the central sequence. Sequence analysis of amplified nucleic acids with nucleic acid arrays has already been reported and could be used, at least over fairly short amplified regions [4]. Another, conceptually simpler, approach might be to select a large number of short random sequences for the chip array, and to compare the binding patterns of generalised amplification products for strains. A range of epidemiologically important organisms could be investigated, culling and substituting sequences that gave invariant or irreproducible binding results. This would eventually provide a generalised typing set with sufficient discrimination and reproducibility for all species. This could be read as a barcode of positive and negative binding results, and it would not be difficult to include a system for electronic download of this information from the diagnostic device to a central epidemiology database.

Although there are many problems to be solved, current molecular and chip technology show the clear promise that a hand-held molecular-chip device capable of rapid detection, susceptibility testing and typing of infectious agents could be developed quite soon and, indeed, preliminary systems are beginning to be publicised. Several aspects of this line of development make it quite different from previous automation in microbiology. First, the device may perform all of the processing required to produce a report. If development proceeds as outlined above, it would effectively be a comprehensively functional microbiology laboratory in a box, in contrast to the limited functions of previous laboratory automation. Second, a hand-held bedside diagnostic device would not have sales limited to microbiology laboratories, but would rapidly find a place in a much larger market comprising all practising physicians and surgeons.

It is possible that such a device might also find a larger niche among the general public of affluent nations, spreading from clear applications for rapid home selfdiagnosis, e.g., in cystic fibrosis patients, to the hypochondriacs. Growing levels of antibiotic resistance, combined with intensive news coverage of outbreaks, may well drive affluent societies to an increasing fear of infection that would encourage this potential market among the general public. Given the potential profits, and the massive investment that our society has made in chip electronics and molecular biology, it would be surprising if development of this concept was neglected, or failed to reach fruition for lack of problemsolving expertise.

The bad news for the diagnostic laboratories is, of course, that the major portion of their work would no longer be required. However, there might well be other serious consequences. Typing investigations and epidemiological surveillance only benefit the individual patient in an indirect way and the desirability of these functions may not be apparent to those involved in development of the device. In a costly rapid development programme it would be easy to dispense with the typing functions described above. If these functions were included, along with a system for downloading the identification, susceptibility and typing data to a central epidemiology database, then this would provide comprehensive information for surveillance of infectious disease on a scale unparalleled by current systems. If, for commercial reasons or neglect, the typing and download functions were omitted, uptake of epidemiological and outbreak data would rely on the interest of individual clinicians, and the efforts of whatever microbiology laboratory service remained after the demise of the laboratory's diagnostic function. 
J.T. MAGEE, J.D. FOX, and S.L.J. STUBBS

Department of Medical Microbiology and Public Health Laboratory, University of Wales College of Medicine, Heath Park, Cardiff CF14 4XW

Corresponding author: Dr J.T. Magee (e-mail: magee@cardiff.ac.uk

\section{References}

1 Lamture JB, Beattie KL, Burke BE et al. Direct detection of nucleic acid hybridization on the surface of a charge coupled device. Nucleic Acids Res 1994; 22: 2121-2125.

2. Kozal MJ, Shah N, Shen $\mathrm{N}$ et al. Extensive polymorphisms observed in HIV-1 clade B protease gene using high-density oligonucleotide arrays. Nat Med 1996; 2: 753-759.

3. Drmanac S, Kita D, Labat I et al. Accurate sequencing by hybridization for DNA diagnostics and individual genomics. Nat Biotechnol 1998; 16: 54-58.

4. Hacia JG, Woski SA, Fidanza J et al. Enhanced high density oligonucleotide array-based sequence analysis using modified nucleoside triphosphates. Nucleic Acids Res 1998; 26: 4975-4982.

5. Livache T, Fouque B, Roget A et al. Polypyrrole DNA chip on a silicon device: example of hepatitis $\mathrm{C}$ virus genotyping. Anal Biochem 1998; 255: 188-194.

6. Chambers J, Angulo A, Amaratunga D et al. DNA microarrays of the complex human cytomegalovirus genome: profiling kinetic class with drug sensitivity of viral gene expression. J Virol 1999; 73: 5757-5766.

7. Gilles PN, Wu DJ, Foster CB, Dillon PJ, Chanock SJ. Single nucleotide polymorphic discrimination by an electronic dot blot assay on semiconductor microchips. Nat Biotechnol 1999; 17: $365-370$.

8. Nilsson P, Larsson A, Lundeberg J, Uhlén M, Nygren P-Å. Mutational scanning of PCR products by subtractive oligonucleo- tide hybridization analysis. Biotechniques 1999; 26: 308-316.

9. Woolley AT, Lao K, Glazer AN, Mathies RA. Capillary electrophoresis chips with integrated electrochemical detection. Anal Chem 1998; 70: 684-688.

10. Asbury CL, van den Engh G. Trapping of DNA in nonuniform oscillating electric fields. Biophys $J$ 1998; 74: 1024-1030.

11. Wilding P, Kricka LJ, Cheng J, Hvichia G, Shoffner MA, Fortina P. Integrated cell isolation and polymerase chain reaction analysis using silicon microfilter chambers. Anal Biochem 1998; 257: 95-100.

12. Cheng J, Sheldon EL, Wu L et al. Preparation and hybridization analysis of DNA/RNA from E. coli on microfabricated bioelectronic chips. Nature Biotechnol 1998; 16: 541-546.

13. Hughes MP, Morgan H, Rixon FJ, Burt JPH, Pethig R Manipulation of herpes simplex virus type 1 by dielectrophoresis. Biochim Biophys Acta 1998; 1425: 119-126.

14. Chan AB, Fox JD. NASBA and other transcription-based amplification methods for research and diagnostic microbiology. Rev Med Microbiol 1999; 10: 185-196.

15. Kopp MU, de Mello AJ, Manz A. Chemical amplification: continuous flow PCR on a chip. Science 1998; 280 1046-1048.

16. Cheng J, Waters LC, Fortina P et al. Degenerate oligonucleotide primed-polymerase chain reaction and capillary electrophoretic analysis of human DNA on microchip-based devices. Anal Biochem 1998; 257: 101-106.

17. Head SR, Parikh K, Rogers Y-H, Bishai W, Goelet P, BoyceJacino MT. Solid-phase sequence scanning for drug resistance detection in tuberculosis. Mol Cell Probes 1999; 13: 81-87.

18. Troesch A, Nguyen H, Miyada CG et al. Mycobacterium species identification and rifampin resistance testing with highdensity DNA probe arrays. J Clin Microbiol 1999; 37: 49-55.

19. Struelens MJ, De Gheldre Y, Deplano A. Comparative and library epidemiological typing systems: outbreak investigations versus surveillance systems. Infect Control Hosp Epidemiol 1998; 19: 565-569. 